\title{
Peak-to-Average Power Ratio Analysis for Golden Coded MIMO Systems
}

\author{
Mohamed L. AMMARI \\ Innov'Com Lab, SUPCOM, \\ Carthage University \\ Tunis, Tunisia
}

\author{
Anis Bdiri \\ Innov'Com Lab, SUPCOM, \\ Carthage University \\ Tunis, Tunisia
}

\begin{abstract}
The combination of the orthogonal frequency-division multiplexing (OFDM) technique and the multiple-input multiple-output (MIMO) architecture has emerged as a promising candidate for future wireless communications. The Space-time block coding (STBC) technique permits to fully take advantage of MIMO-OFDM systems and to effectively improve the channel capacity and the spatial diversity. The Golden Code (GC) is a full-rate and full-diversity, space-time code for $2 \times 2$ MIMO systems. Thanks to its algebraic construction, the GC achieves Diversity-Multiplexing gain trade-off and preserves the mutual information. However, it is well known that STBC-MIMO-OFDM systems are sensitive to the high Peak-to-Average-Power Ratio (PAPR) of the transmitted signals. The purpose of this paper is to analyze the PAPR of golden coded MIMO-OFDM systems. We investigate the effect of the roll-off factor on the PAPR characteristics and analyze the system performances.
\end{abstract}

\section{Keywords}

MIMO, OFDM, PAPR, Golden Code.

\section{INTRODUCTION}

Orthogonal frequency-division multiplexing (OFDM) is an attractive technique for wireless communications over frequency selective fading channels [1]. Its principle is to split the information stream into a large number of low-bit-rate substreams modulating distinct carriers. On the other hand, the Multiple-Input-Multiple-Output (MIMO) systems have been well recognized as effective methods for increasing the channel capacity of the wireless communications [2-4]. The OFDM technique combined with the MIMO architecture allows for both channel capacity increase and performance improvement over frequency selective channels. The spacetime block coding (STBC) is a very useful and efficient transmit scheme that allows exploiting the potential of the MIMO systems [2, 3]. By coding the data stream in both time and spatial domains, the STBC codes permit to maximize the spatial diversity and to improve the system capacity [3].

The first STBC code has been introduced in [3] by Alamouti. Thereafter, several STBC codes have been proposed in the literature [5-7]. In 2004, the STBC-MIMO-OFDM Golden Code (GC) was introduced in [7]. Thanks to its algebraic construction, the GC is a full-rand and a full-rate code for $2 \times 2$ MIMO systems with non-vanishing minimum determinant. In 2006, the GC has been incorporated in the IEEE 802.16 (WiMAX) standard [8]. Authors in [9] have shown that the STBC-MIMO-OFDM combination improves the channel capacity. However, it is well known that the STBC-MIMOOFDM are sensitive to the carrier frequency offset (CFO) and to the Peak-to-Average-Power Ratio (PAPR).
The estimation of the CFO and its effect on the system performances have been addressed in [10].

This paper focuses on the analysis of the PAPR for golden coded MIMO-OFDM systems. As widely discussed in the literature [11-16], one of the major problems of the MIMOOFDM architecture is the high PAPR of the transmitted signal on each antenna. A prohibitively large PAPR seriously reduces the power efficiency of the transmit High Power Amplifier (HPA). In fact, with a high PAPR, the HPA is operating beyond its linear range causing the distortion of the transmitted signal. This distortion generates significant intermodulation and interference between channels [17]. To account for this issue, several techniques have been proposed to mitigate the high PAPR of OFDM signals [13-17]. Among these methods, tone reservation (TR) [18], tone injection (TI) [18], and active constellation extension (ACE) [19] are efficient approaches for PAPR reduction. The principle of these techniques is to modify the constellation for some subcarriers. The clipping method is another used PAPR reduction scheme [20]. Recently, the selected mapping (SLM) technique [15], the partial transmit sequence (PTS) technique [14] and the interleaving technique [21] have been proposed. These techniques generate several alternative signal sequences for the same OFDM signal and select the one with the smallest PAPR.

The effect of the PAPR on the performances of single-carrier MIMO scheme with Alamouti code has been presented in [11]. Statistical analysis of the PAPR in continuous OFDM context is presented in [12]. The statistical description of the PARP is an important key to increase the HPA efficiency. Authors in [22] have presented an upper bound of the PAPR by considering the instantaneous power. The Cumulative Distribution Function (CDF) of the PAPR for OFDM systems is derived in [23]. Authors in [24-25] have considered the MIMO-OFDM system with Alamouti coding and have given the PAPR CDF. In this paper, the CDF for MIMO-OFDM golden coded systems is presented. The PAPR expression is delivered for binary phase shift keying (BPSK) modulated and quadrature phase shift keying (QPSK) systems.

The remainder of this paper is organized as follows. In Section 2, the single-carrier MIMO (SC-MIMO) system with golden code is presented. A theoretical analysis and a closedform expression of the PAPR are then given. In Section 3, we introduce the golden coded MIMO-OFDM system and the SLM scheme and discuss the PAPR proprieties. Section 4 includes numerical results and provides the performance comparison between analytic analysis and simulation curves. Finally, a conclusion is provided in Section 5. 


\section{PAPR OF SC-MIMO SYSTEM WITH GOLDEN CODE}

\subsection{SC-MIMO transmitter}

We consider the $2 \times 2$ Golden coded SC-MIMO system depicted in Fig. 1. We assume that the transmitted binary sequence is BPSK or QPSK modulated. The modulated symbol sequence of length $4 L$ is then coded by a Golden Code with spreading factor 2 . Let us denote by $\boldsymbol{a}(k)=\left[a_{4 k}, a_{4 k+1}, a_{4 k+2}, a_{4 k+3}\right]^{T}$ the Golden encoder input at time $k$. In a matrix form, the Golden codeword is [7]

$$
\boldsymbol{S}(k)=\frac{1}{\sqrt{5}}\left[\begin{array}{cc}
\alpha\left(a_{4 k}+\theta a_{4 k+1}\right) & \alpha\left(a_{4 k+2}+\theta a_{4 k+3}\right) \\
\bar{\alpha}\left(a_{4 k+2}+\bar{\theta} a_{4 k+3}\right) & \bar{\alpha}\left(a_{4 k}+\bar{\theta} a_{4 k+1}\right)
\end{array}\right]
$$

where

$$
\theta=\frac{1+\sqrt{5}}{2}, \quad \bar{\theta}=\frac{1-\sqrt{5}}{2}, \quad \alpha=1+i-i \theta, \quad \bar{\alpha}=1+i-i \bar{\theta}
$$

The ASTC output is digital to analog converted and filtered with the raised-cosine filter. The impulse response of this typical pulse-shaping filter is

$$
g(t)=\operatorname{sinc}\left(\frac{\pi t}{T}\right) \frac{\cos \left(\frac{\beta \pi t}{T}\right)}{1-\left(\frac{2 \beta t}{T}\right)^{2}}
$$

where $\beta$ is the filter roll-off factor.

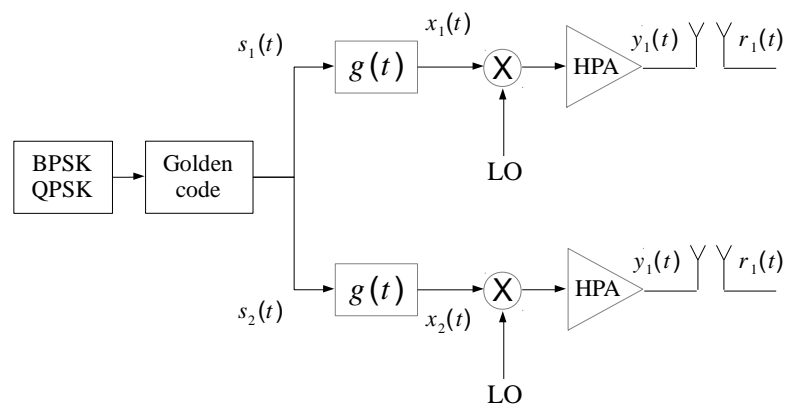

Fig 1: SC-MIMO system with GC

\subsection{PAPR definition}

Let us define the PAPR before and after the raised-cosine filter. The PAPR of the signal $s_{i}(t),(i=1,2)$ is

$$
\operatorname{PAPR}_{b}(i)=\frac{\max _{0 \leq t \leq T}\left|s_{i}(t)\right|^{2}}{E\left[\left|s_{i}(t)\right|^{2}\right]}
$$

By considering the sampled signal, the PAPR before the pulse-shaping filter at the $i$-th antenna is

$$
\operatorname{PAPR}_{b}(i)=\frac{\max _{0 \leq k \leq 4 L}\left|s_{i}(k)\right|^{2}}{E\left[\left|s_{i}(k)\right|^{2}\right]}
$$

In the same way, we define the Peak-to-Mean Envelop Power Ratio (PMEPR) after the raised-cosine filter at the i-th antenna by [11]

$$
\operatorname{PMEPR}_{o}(i)=\frac{\max \left|x_{i}(t)\right|^{2}}{E\left[\left|x_{i}(t)\right|^{2}\right]}
$$

where $x_{i}(t)$ refers to the shaping filter output.

For a QAM modulation, the PMEPR at the raised-cosine filter is upper bound by [11]

$$
\operatorname{PMEPR}_{a}(i) \leq\left(\frac{\max x_{k}}{\sigma_{x}}\right)^{2} \frac{\pi^{2} / 8 \beta}{(1-\beta / 4)}
$$

where $\sigma_{x}$ is the variance of $x_{k}$. Let $\left(x_{i}(t) e^{j 2 \pi f_{c} t}\right)$ be the Radio-Frequency (RF) transmitted signal with carrier frequency $f_{c}$. It was shown in [11] that the PAPR of the passband signal is approximately twice the baseband PMEPR

$$
\left(\operatorname{PAPR}_{a}(i)\right)_{\mathrm{dB}} \approx\left(\operatorname{PMEPR}_{a}(i)\right)_{\mathrm{dB}}+3 \mathrm{~dB}
$$

\subsection{PAPR evaluation for golden coded SC- MIMO system}

Let us consider the golden coded SC-MIMO system depicted in Fig. 1. From (1), we can write

$$
\begin{aligned}
& s_{1}(t)=\sum_{k=0}^{L-1} S_{11}(k) \delta(t-2 k T)-S_{12}(k) \delta(t-(2 k+1) T) \\
& s_{2}(t)=\sum_{k=0}^{L-1} S_{21}(k) \delta(t-2 k T)-S_{22}(k) \delta(t-(2 k+1) T)
\end{aligned}
$$

The raised-cosine filter output can be expressed as

$$
\begin{aligned}
& x_{1}(t)=\sum_{k=0}^{L-1} \boldsymbol{S}_{11}(k) g(t-2 k T)-\boldsymbol{S}_{12}(k) g(t-(2 k+1) T) \\
& x_{2}(t)=\sum_{k=0}^{L-1} \boldsymbol{S}_{21}(k) g(t-2 k T)-\boldsymbol{S}_{22}(k) g(t-(2 k+1) T)
\end{aligned}
$$

Let us assume that the transmit amplifier is linear with unitary gain. The received signal at the first antenna is then

$$
\begin{aligned}
r_{1}(t) & =y_{1}(t) e^{j 2 \pi f_{c t}}+y_{2}(t) e^{j 2 \pi f_{c t} t} \\
& \left.=\sum_{k=0}^{L-1}\left[\left(\boldsymbol{S}_{11}(k)+\boldsymbol{S}_{21}(k)\right) g(t-2 k T)\right]\right]^{j 2 \pi f_{c_{t}}} \\
& +\sum_{k=0}^{L-1}\left[\left(\boldsymbol{S}_{12}(k)+\boldsymbol{S}_{22}(k)\right) g(t-(2 k+1) T)\right] e^{j 22 f_{f t} t}
\end{aligned}
$$

The signal $r_{1}(t)$ can be rewritten as

$$
r_{1}(t)=\sum_{k=0}^{2 L-1} \ell_{k} g(t-k T) e^{j 2 \pi f_{c} t}
$$

where

$$
\ell_{k}= \begin{cases}\boldsymbol{S}_{11}(k / 2)+\boldsymbol{S}_{21}(k / 2), & \text { if } k \text { even } \\ \boldsymbol{S}_{12}((k-1) / 2)+\boldsymbol{S}_{22}((k-1) / 2), & \text { if } k \text { odd }\end{cases}
$$

Using (1), we can express $\ell_{k}$ as

$$
\ell_{k}=\left\{\begin{array}{l}
\frac{\alpha}{\sqrt{5}}\left[a_{2 k}+\theta a_{2 k+1}\right]+\frac{\bar{\alpha}}{\sqrt{5}}\left[a_{2 k+2}+\bar{\theta} a_{2 k+3}\right], \text { if } k \text { even } \\
\frac{\alpha}{\sqrt{5}}\left[a_{2 k+2}+\theta a_{2 k+3}\right]+\frac{\bar{\alpha}}{\sqrt{5}}\left[a_{2 k}+\bar{\theta} a_{2 k+1}\right], \text { if } k \text { odd }
\end{array}\right.
$$

Given (12), we can consider the golden code MIMO system as a SISO case. Thereby, using (7) and (8), we can conclude that the PAPR at the first antenna is upper bounded by

$$
\operatorname{PAPR}_{a}(1) \leq\left(\frac{\max \left|\ell_{k}\right|}{\sigma_{\ell \circ}}\right)^{2} \frac{\pi^{2} / 8 \beta}{(1-\beta / 4)}+3 \mathrm{~dB}
$$




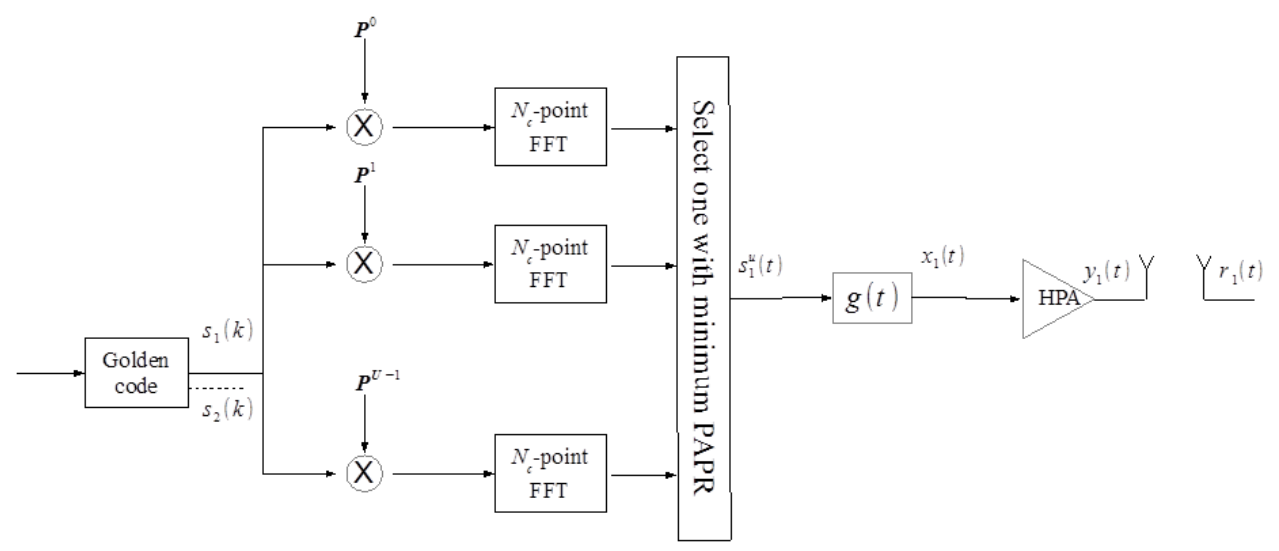

Fig 2: If necessary, the images can be extended both columns

For BPSK modulation, we have $a_{k} \in\{-1,1\}$. By numerical computation, we have $\max \left(\left|\ell_{\ell}\right|\right)=1.94$. On the other hand, as $a_{k}$ and $a_{l}$ are independent for $k \neq l$, we have

$$
\begin{aligned}
\sigma_{y c}^{2} & =E\left[\left|\varphi_{k}\right|^{2}\right] \\
& =\frac{|\alpha|^{2}}{5}\left[E\left(a_{2 k}^{2}\right)+\theta^{2} E\left(a_{2 k+1}^{2}\right)\right]+\frac{|\bar{\alpha}|^{2}}{5}\left[E\left(a_{2 k+2}^{2}\right)+\bar{\theta}^{2} E\left(a_{2 k+3}^{2}\right)\right]
\end{aligned}
$$

if $k$ is even. When $k$ is odd, $E\left[\ell_{k}^{2}\right]$ can be similarly expressed.

Since $E\left(a_{k}^{2}\right)=0.5(-1)^{2}+0.5(1)^{2}=1, \quad$ we have $E\left[l_{k}^{2}\right]=2$. Finally, we get

$$
\left(\frac{\max \ell_{k}}{\sigma_{\ell \circ}}\right)^{2}=1.89
$$

For QPSK modulation, we have $a_{k} \in\{( \pm 1 \pm j) / \sqrt{2}\}$. By numerical computation, we have $\max \left(\ell_{k}\right)=2.75$. Using (17), we have $E\left[\theta_{k}^{2}\right]=2$ and

$$
\left(\frac{\max \ell_{k}}{\sigma_{\ell 6}}\right)^{2}=3.78
$$

The PAPR of BPSK and QPSK modulations at the first antenna, can be evaluated using (14), (16) and (17). The PAPR of $r_{2}(t)$ can be calculated similarly.

\section{PAPR OF MIMO-OFDM SYSTEM WITH GOLDEN CODE}

\subsection{MIMO-OFDM transmitter overview}

For the MIMO-OFDM transmitter, the ASTC output is serialto-parallel-converted and used to modulate $N_{c}$ subcarriers by means of the inverse discrete Fourier transform (IDFT). A cycle prefix (CP) of length $N_{g}$ is then added. In order to avoid the OFDM intersymbol interference, the CP length $N_{g}$ is assumed to be longer than the largest multipath delay spread.
The IDFT output signal at the $i$-th transmit antenna can be written as follows

$$
c_{i}(t)=\sum_{k=0}^{+\infty} \sum_{n=1}^{N_{c}} s_{n, k}^{i} e^{j 2 \pi(n / T) t}, \quad k T \leq t \leq(k+1) T, i=1,2
$$

where $S_{i}(k)=\left[s_{1, k}^{i}, \mathrm{~L} s_{N_{c}, k}^{i}\right]^{T}$ is a vector formed with $N_{c}$ baseband modulated and golden coded symbols and $T=T_{s}+T_{g}$ is the sum of symbol duration $T_{s}$ and the CP length $T_{g}$. The OFDM symbols of length $N_{C}+N_{g}$ are parallel-to-serial-converted and transmitted. Before its transmission, the IDFT output is digital to analog converted and filtered with the raised-cosine filter $g(t)$. In a matrix form, the OFDM symbol at the i-th transmit antenna can be expressed as

$$
\boldsymbol{c}_{i}(k)=\boldsymbol{F}^{H} \boldsymbol{S}_{i}(k)
$$

where $\boldsymbol{c}_{i}(k)=\left[c_{1, k}^{i}, \mathrm{~L}, c_{N_{c}, k}^{i}\right]^{T}$ and $\boldsymbol{F}$ denotes the DFT matrix.

The PAPR of the OFDM symbol $\boldsymbol{s}_{i}(k)$ (before the pulseshaping filter) is

$$
\operatorname{PAPR}_{b}(i)=\frac{\max _{0 \leq n \leq N_{c}}\left|c_{n, k}^{i}\right|^{2}}{E\left[\left|c_{n, k}^{i}\right|^{2}\right]}
$$

As in (7), for a QAM modulation, the PAPR at the raisedcosine filter output is upper bound by [11]

$$
\operatorname{PAPR}_{a}(i) \leq \frac{\max _{0 \leq n \leq N_{c}}\left|c_{n, k}^{i}\right|^{2}}{E\left[\left|c_{n, k}^{i}\right|^{2}\right]} \frac{\pi^{2} / 8 \beta}{(1-\beta / 4)}
$$

The PAPR is a random variable. Therefore, the cumulative distribution function (CDF) and the complementary CDF (CCDF) are often used for characterizing the PAPR. The CCDF of $P A P R_{a}(i)$ is defined as [26]

$$
\operatorname{CCDF}\left(\operatorname{PAPR}_{a}(i)\right)=\operatorname{Pr}\left(\operatorname{PAPR}_{a}(i)>\lambda\right)
$$


Without oversampling, the OFDM samples are uncorrelated. Hence, the CCDF is given by [26]

$$
\operatorname{CCDF}\left(\operatorname{PAPR}_{a}(i)\right)=1-\left(1-e^{\lambda}\right)^{N_{c}}
$$

The PAPR of the MIMO-OFDM system is defined as the worst-case (maximum) PAPR

$$
\operatorname{PAPR}_{a} @ \max _{i=1,2}\left(\operatorname{PAPR}_{a}(i)\right)
$$

For MIMO-OFDM system with $n_{T}$ transmit antennas, we have $N_{c} n_{T}$ samples. The CCDF of the MIMO-OFDM system PAPR is then

$$
\operatorname{CCDF}\left(P A P R_{a}\right)=1-\left(1-e^{\lambda}\right)^{n_{T} N_{c}}
$$

We recall that, in our case, $n_{T}=2$.

\subsection{Review of the SLM Technique}

The SLM is one of the most used PAPR reduction techniques [15]. For this scheme, the transmitter generates several alternative signal sequences for the same OFDM symbol and selects the sequence having the minimum PAPR. A block diagram of OFDM transmitter with the conventional SLM scheme is given in Figure 2.

In order to generate the alternative OFDM signal sequences, $U$ phase rotation vectors $\boldsymbol{P}_{i}^{u}=\left\{P_{i}^{u}(0), \mathrm{L}, P_{i}^{u}\left(N_{c}-1\right)\right\}$ are used. Each element $P_{i}^{u}(n)$ is given by

$$
P_{i}^{u}(n)=\exp \left(j \varphi_{i}^{u}(k)\right), \quad \varphi_{i}^{u}(k) \in[0,2 \pi], \quad \forall 0 \leq u \leq U, \quad i=1,2
$$

An input sequence $\boldsymbol{S}_{i}$ is multiplied by the vector $\boldsymbol{P}_{i}^{u}$ element by element. These $U$ alternative sequences are OFDM modulated by $N_{c}$-point IDFT. Among the resulting sequence $\boldsymbol{c}_{i}^{u},(u=1,2, \mathrm{~L}, U$ and $i=1,2)$, the one with the lowest PAPR $\left(\boldsymbol{c}_{i}^{o p}\right)$ is selected for transmission. We note that in order to properly demodulate the received OFDM signal, the selected $\boldsymbol{P}^{u}$ is transmitted. The sequence $\boldsymbol{c}_{i}^{o p}$ is digital to analog converted and filtered with the raised-cosine filter.

As in (7) and (21), the PAPR at the raised-cosine filter output is upper bound by [11]

$$
\operatorname{PAPR}_{a}(i) \leq \frac{\max _{0 \leq n \leq N_{c}}\left|c_{i}^{o p}\right|^{2}}{E\left[\left|c_{i}^{o p}\right|^{2}\right]} \frac{\pi^{2} / 8 \beta}{(1-\beta / 4)}
$$

The CCDF of the PAPR given by (27) is evaluated as in (25).

\section{SIMULATION RESULTS}

\subsection{PAPR of SC-MIMO system}

The PAPR of the SC-MIMO system as a function of the rolloff factor is given in Fig. 3. Dashed curves result from simulations and solid curves are obtained by equations (14)(17). For the computer simulations, the data length size was fixed to $N=10^{5}$. For $\beta \geq 0.5$, we observe that the analytic bounds are closer to the simulation results. However, for small values $\beta \in[0.1,0.5]$, there is a mismatch between the theoretical bounds and simulation results.

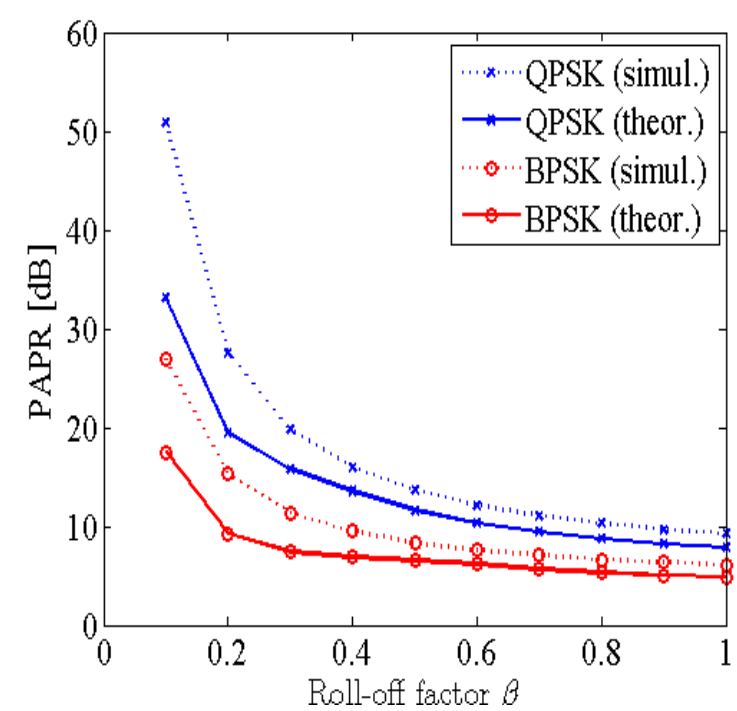

Fig 3: PAPR versus roll-off factor $\beta$ for BPSK and QPSK modulations.

Figure 4 illustrates the CDF of the PAPR for BPSK and QPSK modulations. Curves are obtained by simulation for roll-off factor $\beta=0.7$. For the BPSK modulation, the probability that the PAPR is less than $10 \mathrm{~dB}$ is about $95 \%$. For the QPSK modulation, this probability is about $80 \%$.

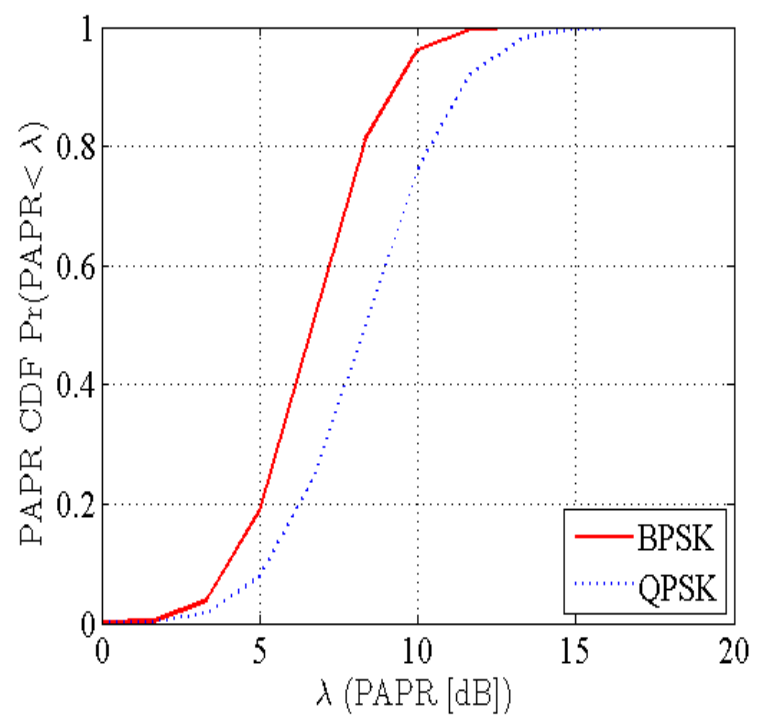

Fig 4: CDF of the PAPR for the golden coded SC-MIMO system.

\subsection{PAPR of MIMO-OFDM system}

For the MIMO-OFDM system, simulations have been carried out on a three tap frequency selective fading channel. The $\mathrm{CP}$ length $N_{g}$ is fixed to $1 / 16$ of the OFDM symbol duration. 


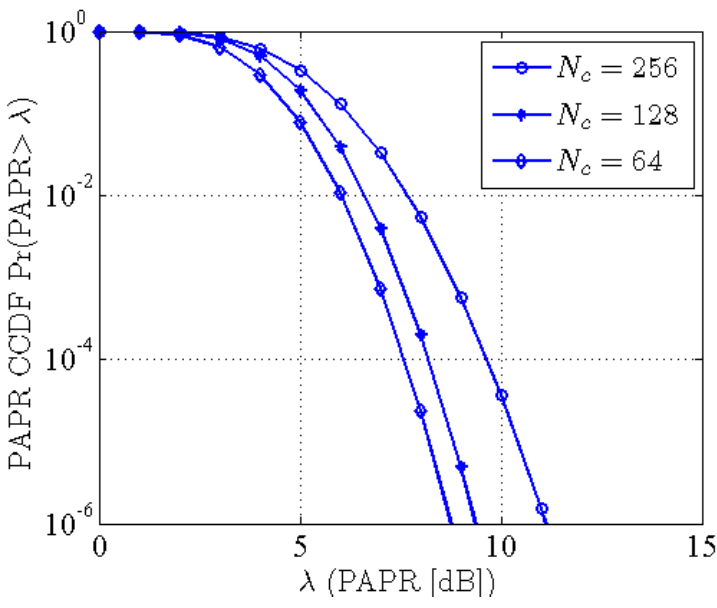

Fig 5: CCDF of the PAPR for the golden coded OFDM system with QPSK modulation and $N_{c} \in\{64,128,256\}$.

Figure 5 shows the CCDF of the PAPR for QPSK modulation and different values of $N_{c}$ (sub-carrier number). In our simulations, the roll-off factor was fixed to $\beta=0.7$ and $10^{5}$ random QPSK symbols are generated to obtain the CCDF of the PAPR. We note that the PAPR is better when the number of subcarriers decreases. As $N_{c}$ decreases from 256 to 64, there is about $2 \mathrm{~dB}$ reduction in the PAPR at a CCDF level of $10^{-4}$. In Fig. 6, we give the CCDF of the PAPR for different values of $\beta$. The transmitted symbols are QPSK modulated and the number of sub-carriers was chosen as $N_{c}=128$. As we can see, the PAPR decreases as the roll-off factor increases. For instance, at the $\mathrm{CCDF}$ of $10^{-4}$, about $3.4 \mathrm{~dB}$ gain is observed when $\beta$ varies from 0.1 to 0.9 .

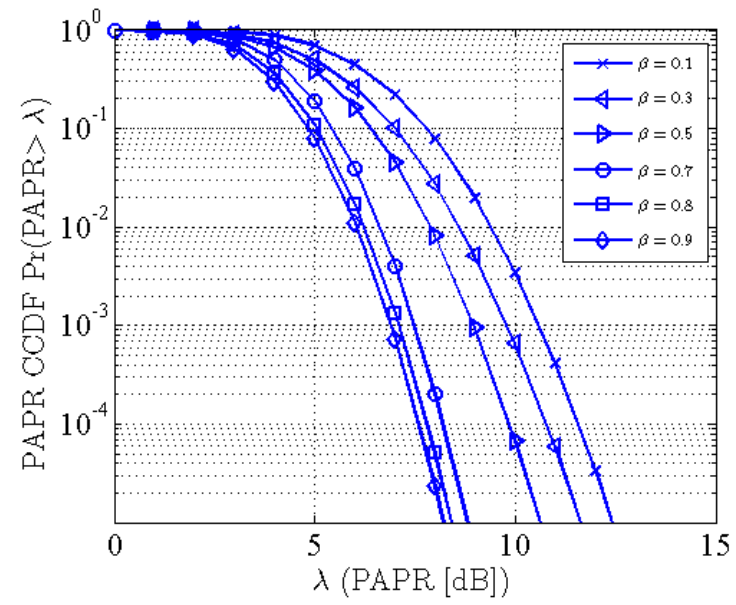

Fig 6: CCDF of the PAPR for the golden coded MIMOOFDM system with different roll-off factors $\left(N_{c}=128\right)$.

Figure 7 compares the CCDF of the PAPR for the golden coded MIMO-OFDM system with and without PAPR reduction. Solid curves correspond to the original system (without PAPR reduction). Dotted curves are obtained by the use of the SLM scheme with $U=20$. The transmitted symbols are QPSK modulated and the number of sub-carriers was chosen as $N_{c}=128$. At the CCDF of $10^{-4}$, the PAPR reduction, achieved by the SLM scheme, is about $3.5 \mathrm{~dB}$ when $\beta=0.1$. However, for $\beta=0.9$, this gain is less important (about $2.2 \mathrm{~dB}$ ).

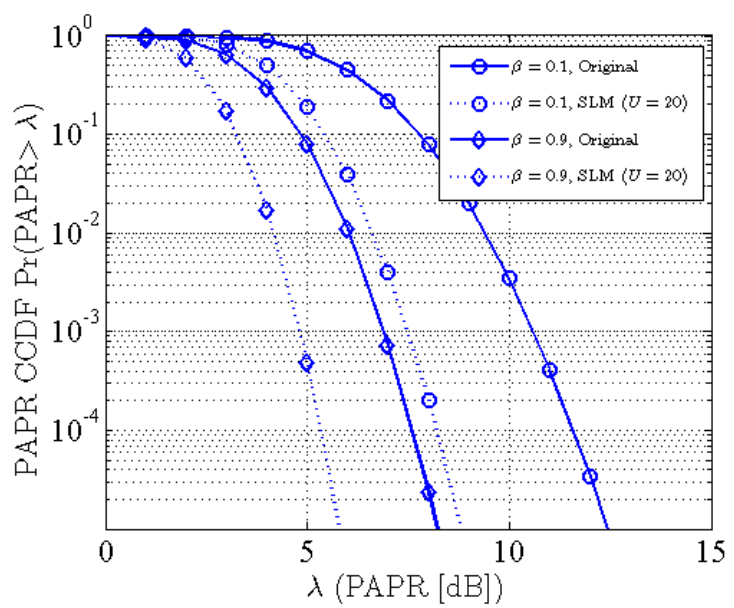

Fig 7: Comparison of the CCDF between the original system and the system with SLM scheme $\left(N_{c}=128\right)$.

The CCDF of the PAPR for $\beta=0.1$ and different values of $U$ are given by Fig 8 . The PAPR of the original system without SLM is also plotted (dotted curves) in Fig. 8. We can see that, when $U$ increases from 4 to 24 , the reduction gain is about $3 \mathrm{~dB}$, at a CCDF of $10^{-4}$. However, when $U$ varies from 20 to 24 , the reduction gain is not noticeable.

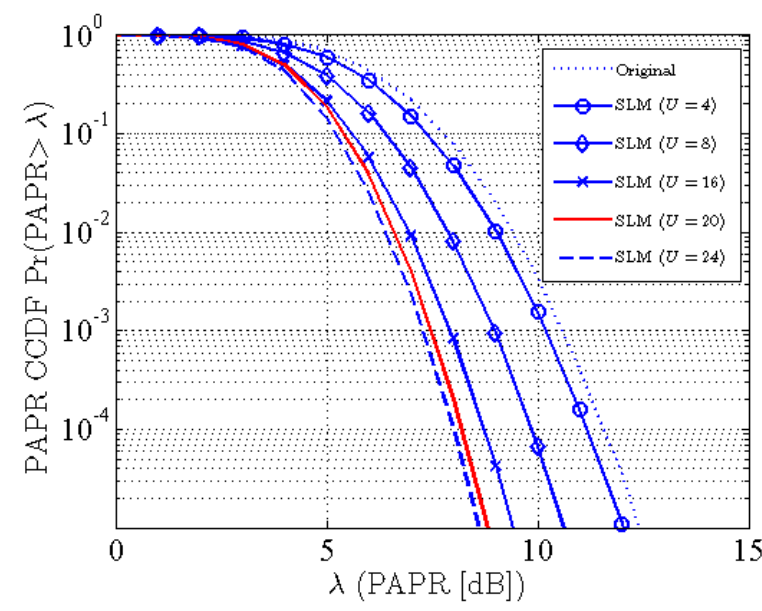

Fig 8: The CCDF of the PAPR for the MIMO-OFDM system with SLM scheme with different values of $U$ (

$$
N_{c}=128 \text { and } \beta=0.1 \text { ). }
$$

Figure 9 compares the BER performance of the golden coded MIMO-OFDM-QPSK systems with and without the SLM scheme. In our simulations, we have assumed that the receiver a has perfect knowledge of the channel and that it is equipped with a maximum-likelihood (ML) detector. The given curves correspond to $N_{c}=128$, a roll-off factor $\beta=0.7$ and $U=20$. 
As we can see, the SLM scheme improves the BER performance. At a BER of $10^{-4}$, the gain due to the PAPR reduction is about $0.8 \mathrm{~dB}$.

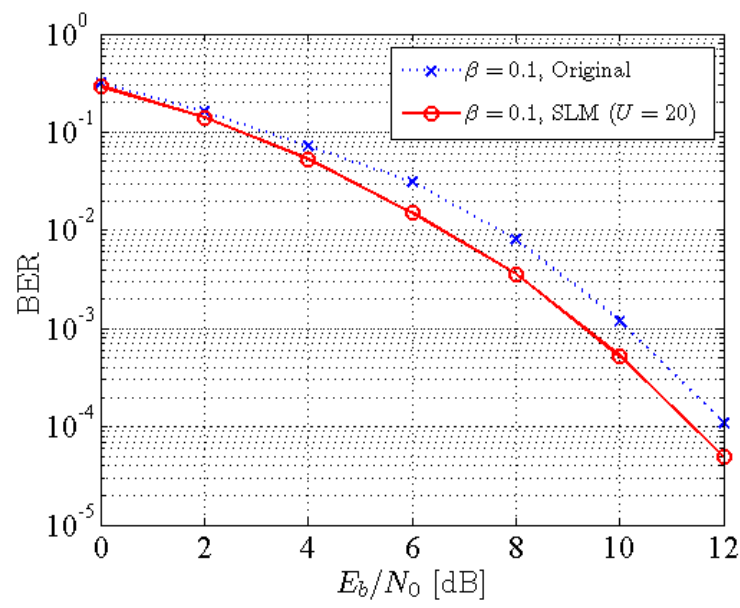

Fig 9: Comparison of the BER for the MIMO-OFDM system with and without SLM scheme ( $N_{c}=128, U=20$ ).

\section{CONCLUSION}

This paper focused on the PAPR analysis of golden coded SCMIMO and MIMO-OFDM systems. For the SC-MIMO system, we have derived a closed-form expression of the PAPR for golden coded symbols. Simulation results showed an agreement between the analytic bound and simulation curves. For the MIMO-OFDM system, we have compared the PAPR of the systems with and without PAPR reduction technique. Simulation results show that the SLM scheme can allow a PAPR reduction of about $3.5 \mathrm{~dB}$. We have noted that, for the SLM scheme, when the number of phase sequences $U$ is larger than 20, the PAPR gain is not noticeable. The BER comparison of MIMO-OFDM-QPSK systems showed that the SLM technique allows an improvement of about $0.8 \mathrm{~dB}$.

\section{REFERENCES}

[1] J. Bingham, "Multicarrier modulation for data transmission: An idea whose time has come", IEEE Commun. Mag., pp. 5-14, May 1990.

[2] G. Foschini, "Layered space-time architecture for wireless communication in a fading environment when using multiple antenna elements", Bell Labs Techn. J., vol. 1, pp. 41-59, Sept. 1996.

[3] S. M. Alamouti, "A simple transmitter diversity scheme for wireless communications", IEEE J. Select. Areas Commun., vol. 16, pp. 1451-1458, Oct. 1998.

[4] V. Tarokh, H. Jafarkhani, and A. R. Calderbank, "Spacetime block codes from orthogonal designs", IEEE Trans. Inform. Theory, vol. 45, pp. 1456-1467, July 1999.

[5] B. A. Sethuraman, B. S. Rajan, and V. Shashidhar, "Fulldiversity, high-rate space-time block codes from division algebras", IEEE Trans. Inf. Theory, vol. 49, pp. 25962616, Oct. 2003
[6] H. El Gamal, and M. O. Damen, "Universal space-time coding", IEEE Trans. Inf. Theory, vol. 49, pp. 10971119, May 2003.

[7] J. C. Rekaya, G. Viterbo, and E. Belfiore, "The golden code: a $2 \times 2$ full-rate space-time code with nonvanishing determinants", IEEE Trans. Inf. Theory, vol. 51, pp.1432-1436, Apr. 2005.

[8] IEEE Standard for local and metropolitan area networks, "Part 16 : Air Interface for Fixed and Mobile Broadband Wireless Access Systems Amendment 2 : Physical and Medium Access Control Layers for Combined Fixed and Mobile Operation in Licensed Bands", Feb. 2006.

[9] A. Bannour, M. L. Ammari, Y. Sun, and R. Bouallegue, "The Capacity Performance of ASTC-MIMO-OFDM System in a Correlated Rayleigh Frequency-Selective Channel", Wireless Personal Communications, vol. 65, pp.1-12, Feb 2012.

[10] A. Bannour, Y. Sun, M. L. Ammari, F. Delestre, and R. Bouallegue, "A Novel Algebraic Carrier Frequency Offset Estimator for ASTC-MIMO-OFDM Systems over a Correlated Frequency Selective Channels", IEEE Trans. Vehic. Techno., vol. 61, pp. 2468-2475, May 2012.

[11] B. Rihawi and Y. Louet, "Peak to Average Power Ratio analysis in MIMO systems", Proc. Info. and Commu. Technologies, 2006.

[12] Y. Louet and S. Hussain, "Peak-to-Mean Envelope Power Ratio Statistical Analysis of Continuous OFDM Signal", Proc. IEEE Vehicular Technology Conf., 2008.

[13] A. E. Jone, T. A. Wilkinson, and S. K. Barton, "Block coding scheme for reduction of peak to mean envelope power ratio of multicarrier transmission schemes", Electron. Lett., vol. 30, pp. 2098-2099, Dec. 1994.

[14] S. H. Muller and J. B. Huber, "OFDM with reduced peak-to-average power ratio by optimum combination of partial transmit sequences", Electron. Lett., vol. 33, pp. 368-369, Feb. 1997.

[15] R. W. Bauml, R. F. H. Fischer, and J. B. Huber, "Reducing the peak-to-average power ratio of multicarrier modulation by selected mapping", Electron. Lett., vol. 32, pp. 2056-1257, Oct. 1996.

[16] L. J. Cimini and N. R. Sollenberger, "Peak-to-average power ratio reduction of an OFDM signal using partial transmit sequences", IEEE Commun. Lett., vol. 4, pp. 86-88, Mar. 2000.

[17] Y.-L. Lee, Y.-H. You, W.-G. Jeon, J.-H. Paik, and H.-K. Song, "Peak-to-Average Power Ratio in MIMO-OFDM Systems Using Selective Mapping", IEEE Commun. Lett., vol. 7, pp. 575-577, Dec. 2003.

[18] J. Tellado and J. M. Cioffi, "Multicarrier Modulation With Low PAR, Application to DSL and Wireless", MA: Kluwer Academic Publisher, 2000.

[19] B. S. Krongold and D. L. Jones, "PAR reduction in OFDM via active constellation extension", IEEE Trans. Broadcast., vol. 49, pp. 258-268, Sep. 2002.

[20] D. Wulich and L. Goldfeld, "Reduction of peak factor in orthogonal multicarrier modulation by amplitude limiting and coding", IEEE Trans. Commun., vol. 47, pp. 18-21, Jan. 1999. 
[21] A. Jayalath and C. Tellambura, "Reducing the peak-toaverage power ratio of orthogonal frequency division multiplexing signal through bit or symbol interleaving", Electron. Lett., vol. 36, pp. 1161-1163, Jun. 2000.

[22] H. Ochiai and H. Imai, "Peak-Power Reduction schemes in OFDM systems: a review", Proc. Intern. Sympos. on Wireless Personal Multimedia Commun., Nov. 1998.

[23] R. Van Nee and A. De Wild, "Reducing the Peak to Average Power ratio of OFDM", Proc. IEEE Vehicular Technology Conf., 1998.
[24] H.-B. Jeon, J.-S. No, and D.-J. Shin, "A Low-Complexity SLM Scheme Using Additive Mapping Sequences for PAPR Reduction of OFDM Signals", IEEE Trans. Broadcast., vol. 57, pp 866-874, Dec. 2011.

[25] B. Rihawi and Y. Louet, "PAPR Reduction Scheme with SOCP for MIMO-OFDM Systems", Inter. J. Commun., Network and System Sciences, pp. 29-35, Jan. 2008.

[26] [26] H. Ochiai and H. Imai, "On the distribution of the peak to average power ratio in OFDM signals", IEEE Trans. Commun., vol. 49, pp. 282-289, Feb. 2001. 